\title{
A NOTE ON BANACH FUNCTION SPACES
}

H. W. ELLIS ${ }^{1}$

1. Introduction. Let $(X \times Y, S \times T, \mu \times \nu)$ denote the completion of the Cartesian product of the $\sigma$-finite and complete measure spaces $(X, S, \mu)$ and $(Y, T, \nu)[5]$. If $\lambda$ is an arbitrary length function on $(X, S, \mu), B$ a Banach space of real valued functions on $Y$ measurable $(T)$, each $f_{x}=f_{x}(y)$ in $L^{\lambda}(B)$ defines a real valued function $f(x, y)$ $=f_{x}(y)$ on $X \times Y$. The purpose of this note is to study the relation between the spaces $L^{\lambda}(B)$ where $B=L^{\Lambda}, \Lambda$ a length function, and the spaces $L^{\lambda \Delta}$ of functions $f(x, y)$, measurable $(S \times T)$, for which $\lambda \Lambda(f)$ $=\lambda\left[\Lambda\left(f_{x}\right)\right]$ defines a norm. Bochner [1] has shown that if $X$ and $Y$ are Euclidean, $\mu$ and $\nu$ Lebesgue measure and $L^{\lambda}$ and $L^{\Lambda}$ are the spaces of summable functions on $X$ and $Y$ then $L^{\lambda \Lambda}$ and $L^{\lambda}\left(L^{\Lambda}\right)$ are equivalent. Our principal result is

Theorem 1.1. $L^{\lambda}\left(L^{\Lambda}\right)$ is always equivalent to a subspace of $L^{\lambda \Delta}$. In order that $L^{\lambda}\left(L^{\Lambda}\right)$ be equivalent to $L^{\lambda \Delta}$ it is necessary and sufficient that either (S) be $\sigma$-atomic $(\lambda)$ or (L9), (L12) and (L13) all hold for $\Lambda$.

2. Terminology. The notation $f(x, y)$ will refer to a real valued function defined on $X \times Y, f_{x}=f_{x}(y)$ to a function on $X$ valued almost everywhere in a space of functions measurable $(T)$ or to an $X$-section of $f(x, y)$. If $Q, Q_{a}$ are subsets of $X \times Y, Q_{x}, Q_{a, x}$ refer to the sections of $Q, Q_{a}$ determined by $x$ [5, p. 141]. By $f_{n}(P) \uparrow f(P)$ is meant $f_{1}(P)$ $\leqq f_{2}(P) \leqq \cdots$ and $f(P)=\sup _{n} f_{n}(P) \leqq \infty$ for all points $P$. Measurability symbols $(S),(T),(S \times T)$ will be omitted when no confusion is likely to result. The definition of simple function will differ from $[5$, p. 84$]$ in that the constants will sometimes be vectors. We shall write $\chi_{E}, \lambda(E)$ as abbreviations for the characteristic function of $E$ and $\lambda\left(\chi_{E}\right)$, respectively, $f_{E}$ for $f \chi_{E}$.

As in [3] $\lambda$ will be called a length function on $(X, S, \mu)$ if for every measurable function $u$ with $0 \leqq u(x) \leqq \infty$ for almost all $x, \lambda(u)$ is defined with $0 \leqq \lambda(u) \leqq \infty$ and satisfies: (L1) $\lambda(u)=0$ if $u(x)=0$ almost everywhere, (L2) $\lambda(u) \leqq \lambda\left(u_{1}\right)$ whenever $u(x) \leqq u_{1}(x)$ for all $x$, (L3) $\lambda\left(u_{1}+u_{2}\right) \leqq \lambda\left(u_{1}\right)+\lambda\left(u_{2}\right)$, (L4) $\lambda(k u)=k \lambda(u)$ for all $k>0$, and (L5) $\lambda(u)=\sup \lambda\left(u_{n}\right)$ if $u_{n} \uparrow u$. In addition we shall sometimes impose some or all of conditions: (L9) Either $X$ is coarse or for every $E$ with $\lambda(E)<\infty, \lambda(e) \rightarrow 0$, whenever $\mu(e) \rightarrow 0, e \subset E[3$, p. 592], (L12)

Received by the editors December 13, 1956 and, in revised form, April 30, 1957.

1 This work was partially supported by the Office of Ordnance Research, U. S. Army, under Contract No. DA-36-034-ORD-1622, Supplemental Agreement No. 2. 
$\lambda(u)<\infty, \epsilon>0$ imply $\lambda\left(u-u_{e}\right)<\epsilon$ for some $e$ with $\lambda(e)<\infty, \quad[3$, p. 592] and (L13) $\lambda(u)<\infty$ implies that $\lambda\left(u-u_{N}\right) \rightarrow 0$ as $N \rightarrow \infty$ where $u_{N}(x)=\min (N, u(x))[3$, p. 577]. We shall suppose throughout that $L^{\lambda}$ and $L^{\Lambda}$ contain at least one element different from zero. We also suppose that $X$ and $Y$ contain no $\lambda, \Lambda$-null sets of positive measure. The theorems remain true when such sets exist, the arguments given below holding after they have been deleted.

If $\mu(E)<\infty, E$ will be called $\sigma$-atomic if the union of the atomic subsets of $E$ (at most countable in number) differs from $E$ by a null set. If every $E \in S$ with $\mu(E)<\infty, 0<\lambda(E)<\infty$ is $\sigma$-atomic we say that $(S)$ is $\sigma$-atomic $(\lambda)$.

The space of functions $f_{x}$, valued in the Banach space $B$, Bochner measurable outside a maximal $\lambda$-null set and with $\lambda\left(f_{x}\right)=\lambda\left(\left|f_{x}\right|\right)<\infty$, will be denoted by $\mathscr{L}^{\lambda}(B)$, the associated Banach space (with points equivalence classes in $\mathscr{L}^{\lambda}(B)$ ) by $L^{\lambda}(B)$. When $\lambda \Lambda(f)=\lambda\left[\Lambda\left(f_{x}\right)\right]$ is defined for every $f(x, y)$, measurable $(S \times T)$, $\mathfrak{2}^{\lambda \Lambda}$ will denote the space of measurable $f(x, y)$ with $\lambda \Lambda(f)<\infty, L^{\lambda \Lambda}$ the associated space.

\section{Spaces of functions measurable $(S \times T)$.}

Theorem 3.1. $L^{\lambda \Lambda}$ is a Banach space.

Proof. Because of [3, Theorem 3.1], we need only show that $\lambda \Lambda$ is a length function when $\lambda$ and $\Lambda$ are length functions (assuming that $\lambda \Lambda(f)$ is defined for every measurable $f$ ). That (L,1)-(L4) hold is easily shown. We verify that (L5) holds for $\lambda \Lambda$. Suppose that $f_{n}(x, y) \uparrow f(x, y)$ where each $f_{n}$ is measurable $(S \times T)$. By $[5,34 . \mathrm{B}, 36.4] f_{n, x}(y)$ is measurable $(T)$ for all $n$ and almost all $x$. If $x$ is such that $f_{n, x}$ is measurable $(T)$ for all $n, \Lambda\left(f_{x}\right)=\sup \Lambda\left(f_{n, x}\right)$ by (L5) for $\Lambda$. There is thus a set $N$ which is a countable collection of null sets, with $\Lambda\left(f_{n, x}\right)$ $\uparrow \Lambda\left(f_{x}\right)$ in $X-N$. (L5) and the fact that $\lambda(g-h)=0$ if $g$ and $h$ differ in a null set then implies that

$$
\lambda \Lambda(f)=\lambda\left[\sup _{n} \Lambda\left(f_{n, x}\right)\right]=\sup _{n} \lambda \Lambda\left(f_{n, x}\right) .
$$

If $\lambda \Lambda$ is to be a length function it must, by definition, be defined for every $f(x, y)$ that is measurable $(S \times T)$. On the other hand if $f(x, y)$ is measurable $(S \times T), f_{x}=f_{x}(y)$ is measurable $(T)$ for almost all $x$ so that $\Lambda\left(f_{x}\right)$ is a function of $x$ defined for almost all $x$. If $\Lambda\left(f_{x}\right)$ is measurable $(S), \lambda \Lambda(f)=\lambda\left[\Lambda\left(f_{x}\right)\right]$ will then be defined for every $f$ measurable $(S \times T)$.

Theorem 3.2. If $f(x, y)$ is measurable $(S \times T), \Lambda\left(f_{x}\right)$ is measurable (S) if: (i) for each $a \geqq 0, E_{a}=\left\{x: \Lambda\left(f_{x}\right)>a\right\}$ differs by a null set from 
$E_{a}^{0}=\left\{x: \nu\left(Q_{a, x}\right)>0\right\}$, where $Q_{a}=\{(x, y):|f(x, y)|>a\}$; or (ii) (L9) holds for $\Lambda$.

Proof. (i) Since $Q_{a}$ is measurable $(S \times T), \nu\left(Q_{a, x}\right)$ is measurable (S) $[5,35 . \mathrm{A}, 36.4]$ so that $E_{a}^{0}$ and, by hypothesis, $E_{a}$ are measurable $(S)$ for every $a$ and therefore $\Lambda\left(f_{x}\right)$ is measurable $(S)$. An example of a length function for which (i) holds for every measurable $f$ is given by

$$
\Lambda[g(y)]=\underset{y \in Y}{\operatorname{ess} . \sup } g(y) .
$$

In the proof of (ii) we shall use

(A) If $E \subset X \times Y$ is measurable $(S \times T)$ then $E \subset \bigcup_{1}^{\infty} A_{i} \times B_{i}$ where $\mu\left(A_{i}\right)<\infty, \nu\left(B_{i}\right)<\infty, i=1,2, \cdots$ and the measurable "rectangles" $A_{i} \times B_{i}$ are disjoint and not empty.

(B) If $\mu \times \nu(E)<\infty, \epsilon>0$, there exists $H=\bigcup_{1}^{n} A_{i} \times B_{i}, \mu\left(A_{i}\right)<\infty$, $\nu\left(B_{i}\right)<\infty, i=1,2, \cdots, n$, with $\mu \times \nu(E \Delta H)<\epsilon$, where $\Delta$ denotes symmetrical difference.

(C) If $f_{n}(x, y) \uparrow f(x, y)$ and $\Lambda\left(f_{n, x}\right)$ is measurable for all $n$, then $\Lambda\left(f_{x}\right)$ is measurable.

The definition of $(X \times Y, S \times T, \mu \times \nu)$ implies (A) and (B) and (L5) for $\Lambda$ and standard measurability theorems give (C).

(ii) We first suppose that $\mu(X)<\infty, \nu(Y)<\infty, \Lambda(Y)<\infty$. If then $f(x, y)=\chi_{Q}$, where $Q$ is measurable $(S \times T)$, there is by (B) a sequence $\left\{H_{n}\right\}$ of finite sums of finite measurable "rectangles" $\bigcup_{i}\left(A_{n i} \times B_{n i}\right)$ $=H_{n}$ with $\mu \times \nu\left(Q \Delta H_{n}\right)$ approaching zero as $n \rightarrow \infty$. Each $\Lambda\left(H_{n}\right)_{x}$ is simple and therefore measurable $(S)$. For a sequence $\epsilon_{n}>0, \epsilon_{n} \rightarrow 0$, let $H_{n}$ be chosen so that $\mu \times \nu\left(Q \Delta H_{n}\right)<\epsilon_{n}^{2}$. If $E_{n}=\left\{x: \nu\left(Q \Delta H_{n}\right)_{x}>\epsilon_{n}\right\}$, $\mu\left(E_{n}\right)<\epsilon_{n}$ since $\mu \times \nu\left(Q \Delta H_{n}\right)=\int_{X}\left(Q \Delta H_{n}\right)_{x} d \mu(x) \quad[5,35 . \mathrm{B}]$. Thus $\left|\Lambda\left(Q_{x}\right)-\Lambda\left(H_{n, x}\right)\right| \leqq \Lambda\left(Q \Delta H_{n}\right)_{x}$ where, by (L9), $\sup \Lambda\left(Q \Delta H_{n}\right)_{x} \rightarrow 0$, $x \in X-E_{n}$, and $\mu\left(E_{n}\right) \rightarrow 0$ as $n \rightarrow \infty$. There is thus a subsequence of $\left\{\Lambda\left(H_{n}\right)_{x}\right\}$ converging almost uniformly to $\Lambda\left(Q_{x}\right)$, which is therefore measurable. If $f(x, y)$ is simple the argument is similar, each set $Q_{i}$ on which $f$ is constant being approximated by sequences of collections of rectangles. The extension to bounded and to arbitrary measurable functions is routine using (C). The proof is then complete when $\mu(X)<\infty, \nu(Y)<\infty, \Lambda(Y)<\infty$.

Suppose that $\nu(Y)<\infty, \Lambda(Y)=\infty$ and let $K$ denote the family of sets $e \in T$, modulo null sets, with $\nu(e)>0, \Lambda(e)<\infty$. If $e_{\alpha}, e_{\beta}$ are in $K$ so is their union. ( $K$ will be empty if there is no function $g(y)$ measurable $(T)$ with $0<\Lambda\left(g_{Y}\right)<\infty$.) $K$ is partially ordered by inclusion. If $\mathscr{N}$ is a nest in $K$, by the Hausdorff maximal principle [7, p. 32], there is a maximal nest $\mathfrak{N}$ in $K$ which contains $\mathscr{N}$. If $e \subset Y-\cup\{M$ : $M \in \mathscr{T}\}$ and $\nu(e)>0$, then $\Lambda(e)=\infty$, since otherwise $\{M \cup e\}$ would 
be a nest properly containing $\mathfrak{T}$. For $i=1,2, \ldots$ let $Y_{i}$ be an arbitary set in $\mathfrak{M}$ with $\nu\left(Y_{i}\right)>\left(1-1 / 2^{i}\right) \sup _{M \in \mathfrak{T}} \nu(M)$. Then $\nu\left(U Y_{i}\right)$ $=\sup \nu(M) \leqq \nu(Y)$ and

(3.1) $Y=Y_{0}+\bigcup_{1}^{\infty} Y_{i}, \quad \Lambda(e)=\infty$ if $e \subset Y_{0}, \nu(e)>0 ; \quad \Lambda\left(Y_{i}\right)<\infty$, $i=1,2, \cdots$ (cf. $[8, \S \S 2,3])$. If $f(x, y)$ is measurable $(S \times T),(\mathrm{A})$ and the $\sigma$-finiteness of $\nu$ permit us to assume that $f(x, y)=0$ for $y$ outside $Y=\bigcup_{1}^{\infty} B_{i}$ with $\nu\left(B_{i}\right)<\infty$ and combining the decompositions (3.1) for all $B_{i}$ shows that (3.1) holds for $Y$. Since $\mu$ is also $\sigma$-finite we can assume that $f(x, y)$ vanishes outside $X=\bigcup_{1}^{\infty} X_{i}$ with $\mu\left(X_{i}\right)<\infty$ and that, for $H_{n}=\mathrm{U}_{1}^{n} X_{i} \times Y_{i}, Y^{\prime}=Y-Y_{0}, f_{H_{n}} \uparrow f_{X \times Y^{\prime}}$ as $n \rightarrow \infty$ so that $f_{X \times Y}$ is measurable by the first part of the proof and (C). Finally if $E_{x}=\left\{y \in Y_{0}: f(x, y)>0\right\}, \Lambda\left(f_{x}\right)=\infty$ if $\nu\left(E_{x}\right)>0,=\Lambda\left(f_{X \times Y^{\prime}}\right)$ if $\nu\left(E_{x}\right)$ $=0$. Since $\left\{x: \nu\left(E_{x}\right)>0\right\}$ is measurable, $\Lambda\left(f_{x}\right)$ is measurable.

REMARK. If $\lambda$ and $\Lambda$ determine $L^{1}$ norms, Theorem 3.2 is part of the Fubini theorem.

If $f(x, y)$ is measurable $(S \times T), f_{x}$ is valued almost everywhere in the space of real functions measurable $(T)$. If $f \in \mathcal{L}^{\lambda \Lambda}, f_{x}$ is valued almost everywhere in $\mathscr{L}^{\Lambda}, \lambda\left(f_{x}\right)=\lambda \Lambda(f)<\infty$ and $f_{x} \in \mathcal{L}^{\lambda}\left(L^{\Lambda}\right)$ if and only if $f_{x}$ is Bochner measurable.

L.EMMA 3.1. In order that, for every $f(x, y) \in \mathcal{L}^{\lambda \Lambda}, f_{x} \in \mathcal{L}^{\lambda}\left(L^{\Lambda}\right)$ it is necessary and sufficient that either (i) $(S)$ is $\sigma$-atomic $(\lambda)$ or (ii) satisfies (I.9), (L12) and (L13).

Proof. Assume that (i) holds. The definition of $(X \times Y, S \times T$, $\mu \times \nu)$ implies that, if $f(x, y)$ is measurable $(S \times T)$ and $S_{0} \in S$ is atomic, there is a function $\bar{f}(x, y)$ with $\bar{f}_{x}$ constant in $S_{0}$ and with $\mu \times \nu\left(S_{1}\right)=0$ where $S_{1}=\{(x, y): f(x, y) \neq \bar{f}(x, y)\}$ (cf. $[5,16.4$, p. 72]).

L.et $f \in \mathfrak{L}^{\lambda \Lambda}$ and suppose that $\mu(x)<\infty$. If $X_{n}=\left\{x: \Lambda\left(f_{x}\right)>1 / n\right\}$, then $X_{0}=\left\{x: \Lambda\left(f_{x}\right)>0\right\}=\bigcup_{1}^{\infty} X_{n}, \mu(N)=0$ where $N=\left\{x: \Lambda\left(f_{x}\right)=\infty\right\}$, and $\mu\left(X_{0}-X_{n}\right) \rightarrow 0$ as $n \rightarrow \infty$. For each $n$,

$$
0 \leqq \Lambda\left(X_{n}\right) \leqq \Lambda\left(n f_{x}\right) \leqq n \Lambda\left(f_{x}\right)<\infty, \quad x \in X_{0}-N .
$$

Since $X$ is $\sigma$-atomic $(\lambda)$ each $X_{n}=\cup_{i=1}^{\infty} A_{n i}+N_{n}$ where each $A_{n i}$ is atomic and $\mu\left(N_{n}\right)=0$. Then $f_{x}$ is equivalent $(S \times T)$ to $\bar{f}_{x}$ which is constant on each $A_{n i}$. The proof that $\bar{f}_{x}$ and $f_{x}$ are Bochner measurable on $X$ is then trivial. The extension to an arbitrary $X$ is then a consequence of the $\sigma$-finiteness of $\mu$.

We next show that (ii) is sufficient. Since $\mu$ is $\sigma$-finite we can assume that $\mu(X)<\infty$. In the first part of the proof of Theorem 3.2 (ii) each $\chi_{H_{n}, x}$ is simple and it follows easily that $\chi_{Q_{x}}$ is Bochner measurable. 
The extension to $f_{x}$ corresponding to a bounded, measurable $f(x, y)$ is easy. If $f(x, y) \in \mathfrak{L}^{\lambda \Lambda}$ is not bounded, $\lambda \Lambda(f)<\infty$ implies that $\Lambda\left(f_{x}\right)$ $<\infty$ outside a $\mu$-null set $X_{0} \subset X$. (L13) then implies that $\Lambda\left(f-f_{N}\right)_{x} \rightarrow 0$ as $N \rightarrow \infty, x \in X_{0}$. For $\epsilon>0$ let $E_{N}=\left\{x: \Lambda\left(f-f_{N}\right)_{x}>\epsilon\right\}$. For $N$ sufficiently large $\mu\left(E_{N}\right)<\epsilon$ since $\mu(X)<\infty$. This, with the Bochner measurability of all the $f_{N}$ implies that $f$ is Bochner measurable when $\nu(Y)<\infty, \Lambda(Y)<\infty$. In the general case we can assume from (A) that $f(x, y)=0$ outside $\bigcup_{1}^{\infty} B_{i}$ where $\nu\left(B_{i}\right)<\infty, \Lambda\left(B_{i}\right)<\infty, i=1,2, \cdots$. Then $f_{x}^{m}=f_{x} \chi \cup_{1^{m} B_{i}}$ is Bochner measurable for every $m$. For each $x$ outside a $\lambda$-null set, where $e$ and $N$ are as in (L12) and (L13),

$$
\begin{aligned}
\Lambda\left(f_{x}-f_{x}^{m}\right) \leqq & \Lambda\left(f_{x}-f_{N, x}\right)+\Lambda\left(f_{N, x}^{m}-f_{x}^{m}\right)+\Lambda\left(f_{N, x}-f_{N, x} \chi_{e}\right) \\
& +\Lambda\left(f_{N, x}-f_{N, x}^{m}\right)_{e}+\Lambda\left(f_{N, x}^{m}-f_{N, x}^{m} \chi_{e}\right) \\
\leqq & 2 \Lambda\left(f_{x}-f_{N, x}\right)+2 \Lambda\left(f_{N, x}-f_{N, x} \chi_{e}\right)+N \Lambda\left(e \bigcup_{m}^{\infty} B_{i}\right),
\end{aligned}
$$

which can be made arbitrarily small by choice of $N, e$ and $m$, using (L12) and (L13). That $f_{x}$ is Bochner measurable then follows easily as in the preceding paragraph.

Finally we show that at least one of (i), (ii) is necessary to ensure that, for every $g(x, y) \in \mathcal{L}^{\lambda \Lambda}, g_{x}$ is Bochner measurable. Suppose that $S$ contains a set $S_{1}$ with $\mu\left(S_{1}\right)<\infty, 0<\lambda\left(S_{1}\right)<\infty$ and suppose that $S_{1}$ contains no atoms. Then $S$ contains a sequence $S_{n}$ of sets satisfying $S_{1}=S_{2} \cup S_{3}, S_{2}=S_{4} \cup S_{5}$ and in general $S_{i}=S_{j} \cup S_{j+1}$ for some $j>i$, where $\mu\left(S_{i}\right)>0, i=1,2, \cdots$ and $\mu\left(S_{i}\right) \rightarrow 0$ as $i \rightarrow \infty$ (cf. [4, §2]). Suppose that there is a non-negative function $f(y) \in L^{\Lambda}$, a number $\delta>0$, and a collection of disjoint sets $\left\{T_{i}\right\}$ in $T$ with $\Lambda\left(f_{T_{i}}\right)>\delta, i=1,2, \cdots$. Define $g(x, y)=f_{T_{i}}(y)=f \chi_{T_{i}}$ for all $x \in S_{i}, i=1,2, \cdots, g(x, y)=0$ elsewhere. Then clearly $g(x, y)$ is measurable $(S \times T)$ and, since $\Lambda\left(g_{x}\right) \leqq \Lambda\left(f_{x}\right)$ for all $x \in S_{1}, \Lambda\left(g_{x}\right)=0$ elsewhere, $\lambda \Lambda(g) \leqq \Lambda(f) \lambda\left(S_{1}\right)<\infty$ so that $g \in \mathfrak{L}^{\lambda \Lambda}$. If for some $n, S_{n}$ contains $x$ but not $x^{\prime} \in S_{1}$,

$$
\left|g_{x}(y)-g_{x^{\prime}}(y)\right| \geqq f_{T_{i}}(y), \quad \Lambda\left(g_{x}-g_{x^{\prime}}\right) \geqq \Lambda\left(f_{T_{i}}\right)>\delta .
$$

Thus if $x$ is fixed and $\left\{S_{n_{i}}\right\}$ is the subsequence of $\left\{S_{n}\right\}$ containing $x$, the set of points $x^{\prime}$ for which $\Lambda\left(g_{x}-g_{x^{\prime}}\right)<\delta$ is contained in $\cap S_{n_{i}}$ and therefore has measure zero. If $\bar{g}_{x}$ is an arbitrary simple function on $X, \Lambda\left(g_{x}-\bar{g}_{x}\right)>\delta / 2$ for almost all $x \in S_{1}$ so that $g_{x}$ cannot be Bochner measurable. The argument of [4, Lemma 3.2] shows that there always exists such a function $f(y) \in L^{\Delta}, \delta>0$ and $\left\{T_{i}\right\}$ if one of (L9), (L12) and (L13) fails to hold so that the above construction gives a counterexample if (i) and one of (L9), (L12) and (L13) fail to hold. 
4. The equivalence of the spaces $L^{\lambda \Lambda}$ and $L^{\lambda}\left(L^{\Delta}\right)$. Where $\mu, \nu$ denote Lebesgue measure on $(0,1)=X=Y$, Sierpinski [9] (using the axiom of choice) has constructed a plane set $Q$ having at most two points in common with every parallel to the axes and not measurable $(S \times T)$. For the function $\chi_{Q}, \Lambda\left(Q_{x}\right)=0$ for all $x$ so that $\chi_{Q_{x}}$ is Bochner measurable and in $\mathscr{L}^{\lambda}\left(L^{\Lambda}\right)$ but not in $\mathscr{L}^{\lambda \Lambda}$ since it is not measurable. This example shows that the statement of Theorem I in [1] is not precise. We note that when the conditions of Lemma 3.1 are not satisfied (for example if $L^{\Lambda}=L^{\infty}$, and $x, y, \mu, \nu$ are as in this paragraph) $\mathfrak{L}^{\lambda \Lambda}$ and $\mathscr{L}^{\lambda}\left(L^{\Lambda}\right)$ can each contain elements with the correspond ing element not in the other. We note that for Sierpinski's example there is a function (namely $g_{x} \equiv 0$ ) equivalent to $f_{x}$ in $L^{\lambda}\left(L^{\Lambda}\right)$ with $g(x, y)$ measurable $(S \times T)$. That this is always the case is shown by the following lemma which generalizes part of Bochner's Theorem I $[1]$.

LEMmA 4.1. If $f_{x} \in L^{\lambda}\left(L^{\Lambda}\right)$ then $\hat{f}_{x}$, the equivalence class of $f_{x}$, contains $g_{x}$ with $g(x, y) \in L^{\lambda \Lambda}$.

Proof. First suppose that $\mu(X)<\infty, \lambda(X)<\infty$. For $\epsilon>0$ the Bochner measurability of $f_{x}$ implies the existence of a set $E_{1} \subset X=E_{0}$ with $\mu\left(E_{1}\right)<\epsilon$ and a sequence of simple functions $f_{x}^{i}$ valued in $L^{\Lambda}$ with $\Lambda\left(f_{x}-f_{x}^{i}\right) \rightarrow 0$ uniformly in $E_{0}-E_{1}$. Each $f^{i}(x, y) \in L^{\lambda \Lambda}$ and the $f^{i}$, restricted to $E_{0}-E_{1}$, form a Cauchy sequence in $L^{\lambda \Lambda}$ and define a function $g_{1}(x, y) \in L^{\lambda \Lambda}$, vanishing in $E_{1} \times Y$, with $\lambda\left(f_{x}-g_{1, x}\right)_{E_{0}-E_{1}}=0$. Replacing $E_{i}$ by $E_{i+1}, i=1,2, \cdots$, there is a sequence of sets $E_{i}$ with $E_{i} \subset E_{i+1}, \mu \bigcup_{0}^{\infty}\left(E_{i}-E_{i+1}\right)=\mu(X)$, and a corresponding sequence of functions $g_{i}(x, y) \in L^{\lambda \Lambda}$, vanishing outside $E_{i-1}-E_{i}$ with

$$
\lambda\left(f_{x}-g_{i, x}\right)_{E_{i-1} E_{i}}=0 .
$$

Set $g(x, y)=\sum_{1}^{\infty} g_{i}(x, y)$. Then $g(x, y) \in L^{\lambda \Lambda}, g_{x} \in \mathcal{L}^{\lambda}\left(L^{\Lambda}\right)$ and, since $\left(f_{x}-g_{x}\right) \cup_{1}^{n} E_{i}-E_{i+i} \uparrow\left(f_{x}-g_{x}\right)$ outside a null set, (4.1) and (L5) for $\lambda$ show that $g_{x} \in \hat{f}_{x}$.

In the general case the set $X_{0}=\left\{x: \Lambda\left(f_{x}\right)>0\right\}$ is measurable $(S)$. If $X_{n}=\left\{x: \Lambda\left(f_{x}\right)>1 / n\right\}, X_{0}=\cup_{1}^{\infty} X_{n}$ and $\lambda\left(X_{n}\right)<\infty, n=1,2, \cdots$. Since $X_{0}$ is $\sigma$-finite we can assume that $\mu\left(X_{n}\right)<\infty$ and that the $X_{n}$ are disjoint. An argument similar to that given in the first part then completes the proof.

Lemmas 3.1 and 4.1 combine to give Theorem 1.1. Theorem 1.1 has the following corollaries.

Corollary 1. If $L^{\Lambda}$ is separable or reflexive, $L^{\lambda \Lambda}$ is equivalent to $L^{\lambda}\left(L^{\Lambda}\right)$. 
Corollary 2. $L^{\lambda \Lambda}$ is separable (reflexive) if and only if $L^{\lambda}$ and $L^{\Lambda}$ are separable (reflexive).

If $L^{\wedge}$ is separable (L9), (L12) and (L13) hold by [4, Lemma 3.2]. If $L^{\Lambda}$ is reflexive they hold by [6, p. 206]. If $L^{\lambda \Lambda}$ is separable (reflexive), $L^{\Lambda}$ is separable (reflexive) since it is isometric to a subspace of $L^{\lambda \Delta}$. Then Corollary 1, [4, Lemma 3.2] and [6, Theorem 1.1] give Corollary 2.

The basis problem for the spaces $L^{\lambda \Lambda}$ is equivalent to the basis problem for the spaces $L^{\lambda}\left(L^{\Lambda}\right)$ and is therefore partially solved by [2]. That the results of [4] do not apply directly to $L^{\lambda \Lambda}$ is shown by the fact that even when both $\lambda$ and $\Lambda$ are levelling (i.e. have property (L8) of length functions [3]) $\lambda \Lambda$ need not be leveling or even have the weaker property (3.4) of [4]. This is shown by the following elementary example. Let $X=Y=(0, \infty)$, let $S, T$ be the Lebesgue measurable sets, $\mu$ and $\nu$ Lebesgue measure on $(0, \infty)$ and let $L^{\lambda}=L^{2}$, $L^{\Lambda}=L^{1}$. Let $R_{n}=\{(x, y): n-1<x \leqq n ; n-1<y \leqq n\}, R_{n}^{\prime}=\{(x, y)$ : $0<x<1, n-1<y \leqq n\}$. Let $f_{k}(x, y)$ denote the characteristic function of $\bigcup_{1}^{k} R_{n}, A f_{k}$ the average of $f_{k}$ over $R_{n} \cup R_{n}^{\prime}$ in $R_{n} \cup R_{n}^{\prime}, n=1,2, \cdots$; $=f_{k}$ elsewhere. Then every $f_{k} \in L^{\lambda \Lambda}$ but actual computation shows that $\lambda \Lambda\left(A f_{k}\right) / \lambda \Lambda\left(f_{k}\right)>k^{1 / 2} / 2$.

\section{REFERENCES}

1. S. Bochner, Eine Bemerkung zum Satz von Fubini, Fund. Math. vol. 20 (1933) pp. $277-280$.

2. H. W. Ellis, On the basis problem for vector valued function spaces, Canadian Journal of Mathematics vol. 8 (1956) pp. 412-422.

3. H. W. Ellis and Israel Halperin, Function spaces determined by a levelling length function, Canadian Journal of Mathematics vol. 5 (1953) pp. 576-592.

4. - Haar functions and the basis problem for Banach spaces, J. London Math. Soc. vol. 31 (1956) pp. 28-39.

5. P. R. Halmos, Measure theory, New York, 1950.

6. Israel Halperin, Reflexitivity in the $L^{\lambda}$-function spaces, Duke Math. J. vol. 21 (1954) pp. 205-208.

7. J. L. Kelley, General topology, New York, 1955.

8. W. A. J. Luxemburg and A. C. Zaanen, Some remarks on Banach function spaces, Neder. Akad. Wetensch. vol. 59 (Indagationes Math. 18) (1956) pp. 110-119.

9. W. Sierpinski, Sur un problème concernant les ensembles measurables superficiellement, Fund. Math. vol. 1 (1920) pp. 112-115.

INSTITUTE FOR ADVANCED StUdy 